\title{
Application of the Gel'fand Matrix Method to the Missing Label Problem in Classical Kinematical Lie Algebras
}

\author{
Rutwig CAMPOAMOR-STURSBERG
}

Departamento Geometría y Topología, Fac. CC. Matemáticas U.C.M., Plaza de Ciencias 3, E-28040 Madrid, Spain

E-mail: rutwig@mat.ucm.es

Received November 06, 2005, in final form February 14, 2006; Published online February 28, 2006

Original article is available at http://www.emis.de/journals/SIGMA/2006/Paper028/

\begin{abstract}
We briefly review a matrix based method to compute the Casimir operators of Lie algebras, mainly certain type of contractions of simple Lie algebras. The versatility of the method is illustrated by constructing matrices whose characteristic polynomials provide the invariants of the kinematical algebras in (3+1)-dimensions. Moreover it is shown, also for kinematical algebras, how some reductions on these matrices are useful for determining the missing operators in the missing label problem (MLP).
\end{abstract}

Key words: Casimir operator; characteristic polynomial; Lie algebra; missing label; kinematical group

2000 Mathematics Subject Classification: 17B05; 81R05

\section{Introduction}

Casimir operators of Lie algebras were originally introduced in the frame of representation theory of semisimple algebras, and were soon recognized as a powerful tool for the structural analysis. They were determined by means of the universal enveloping algebra of a Lie algebra, and successive applications of Lie groups and algebras to other mathematical and physical problems led to alternative methods to compute these invariants, like the orbit method or the formulation in terms of differential equations, which allow to generalize the concept of invariant functions on Lie algebras beyond the pure algebraic frame.

In 1950 I.M. Gel'fand [1] showed how to use the generic matrix of standard representation of orthogonal groups to determine the Casimir operators of the algebra by means of characteristic polynomials. More specifically, for the pseudo-orthogonal Lie algebra $\mathfrak{s o}(p, q)(N=p+q)$ given by the $\frac{1}{2} N(N-1)$ operators $E_{\mu \nu}=-E_{\nu \mu}$ and having brackets:

$$
\left[E_{\mu \nu}, E_{\lambda \sigma}\right]=g_{\mu \lambda} E_{\nu \sigma}+g_{\mu \sigma} E_{\lambda \nu}-g_{\nu \lambda} E_{\mu \sigma}-g_{\nu \sigma} E_{\lambda \mu}
$$

where $g=\operatorname{diag}(1, \ldots, 1,-1, \ldots,-1)$, the Casimir operators were obtained with the formula [1]:

$$
P(\lambda)=\left|M_{p, q}-\lambda \operatorname{Id}_{N}\right|=\lambda^{N}+\sum_{k=1}^{N} C_{k} \lambda^{N-k},
$$


$M_{p, q}$ being the matrix

$$
M_{p, q}=\left(\begin{array}{ccccc}
0 & \cdots & -g_{j j} e_{1 j} & \cdots & -g_{N N} e_{1 N} \\
\vdots & & \vdots & & \vdots \\
e_{1 j} & \cdots & 0 & \cdots & -g_{N N} e_{j N} \\
\vdots & & \vdots & & \vdots \\
e_{1 N} & \cdots & g_{j j} e_{j N} & \cdots & 0
\end{array}\right)
$$

and $\operatorname{Id}_{N}$ the identity matrix of order $N$. Once symmetrized, the functions $C_{k}$ provide the classical operators in the enveloping algebra of $\mathfrak{s o}(p, q)$. This direct matrix approach seems natural, taking into account that the eigenvalues of Casimir operators are essential for the labelling of representations of the Lie algebra. By application of the classical theory of semisimple Lie algebras, similar formulae can be developed for other semisimple Lie algebras [2]. It is natural to ask whether the essence of the Gel'fand method can be generalized to other non-semisimple Lie algebras, by means of extended matrices that possibly correspond to some representation of the algebra. The two main interesting cases are:

1. Generalized Inönü-Wigner contractions of (semi)simple Lie algebras. We have inhomogeneous algebras as a special case.

2. Semidirect products of simple and Heisenberg Lie algebras.

The method usually employed to determine the invariants of a Lie algebra consists in solving of a system of partial differential equations (PDEs) related to a realization of the Lie algebra as differential operators $[3,4]$. If $\left\{X_{1}, \ldots, X_{n}\right\}$ is a basis of $\mathfrak{g}$ and $\left\{C_{i j}^{k}\right\}$ is the structure tensor, the realization of $\mathfrak{g}$ in the space $C^{\infty}\left(\mathfrak{g}^{*}\right)$ is given by:

$$
\widehat{X}_{i}=-C_{i j}^{k} x_{k} \partial_{x_{j}},
$$

where $\left[X_{i}, X_{j}\right]=C_{i j}^{k} X_{k}(1 \leq i<j \leq n, 1 \leq k \leq n),\left\{x_{1}, \ldots, x_{n}\right\}$ is the corresponding dual basis, $\mathfrak{g}^{*}$ being the dual space to $\mathfrak{g}$. Invariants of the coadjoint representation are functions on the generators $F\left(X_{1}, \ldots, X_{n}\right)$ of $\mathfrak{g}$ such that $\left[X_{i}, F\left(X_{1}, \ldots, X_{n}\right)\right]=0$, and are determined by solving the system of PDEs:

$$
\widehat{X}_{i} F\left(x_{1}, \ldots, x_{n}\right)=-C_{i j}^{k} x_{k} \frac{\partial F}{\partial x_{j}}\left(x_{1}, \ldots, x_{n}\right)=0, \quad 1 \leq i \leq n .
$$

Classical Casimir operators are recovered with replacing the variables $x_{i}$ by the corresponding generator $X_{i}$ (possibly after symmetrizing). The cardinal $\mathcal{N}(\mathfrak{g})$ of a maximal set of independent solutions is described in terms of the following formula:

$$
\mathcal{N}(\mathfrak{g})=\operatorname{dim} \mathfrak{g}-\operatorname{rank} A(\mathfrak{g}),
$$

where $A(\mathfrak{g})$ is the matrix representing the commutator table of $\mathfrak{g}$ over a given basis, i.e.,

$$
A(\mathfrak{g})=\left(C_{i j}^{k} x_{k}\right) .
$$

In this work we briefly review some recent work on matrix procedures that generalize the Gel'fand formula for various types of Lie algebras, specifically inhomogeneous Lie algebras. Applications to the computation of missing label operators are also given.

\section{Contractions of simple Lie algebras}

Let $\mathfrak{g}$ be a simple Lie algebra of rank $k$ such that the Casimir operators of $\mathfrak{g}$ are given by the characteristic polynomial $P(T)$ of a matrix

$$
A=\sum_{i} \Gamma_{1}\left(X_{i}\right) x_{i}
$$


where $\Gamma_{1}$ is equivalent to the $N$-dimensional standard representation of $\mathfrak{g}$. Suppose further that the Lie algebra $\mathfrak{g}^{\prime}$ is a contraction of $\mathfrak{g}$ and that $\mathcal{N}(\mathfrak{g})=\mathcal{N}\left(\mathfrak{g}^{\prime}\right)$. To compute the Casimir operators of $\mathfrak{g}^{\prime}$ using a matrix extension of $A$, the following procedure by steps has been proposed [5]:

1. Take the matrix $A=\sum_{i} \Gamma_{1}\left(X_{i}\right) x_{i}$.

2. Let $\Psi_{\epsilon}$ be the automorphism giving the contraction ${ }^{1}$ and consider the matrix $A^{\prime}=$ $\left.\sum_{i} \Gamma_{1}\left(X_{i}^{\prime}\right)\right) x_{i}^{\prime}$ giving the invariants of $\mathfrak{g}$ over the transformed basis $\left\{X_{i}^{\prime}:=\Psi_{\epsilon}\left(X_{i}\right)\right\}$.

3. Let $P_{\epsilon}(T):=\left|A^{\prime}-T \operatorname{Id}_{N}\right|$ and $\alpha:=\operatorname{deg}_{\epsilon} P_{\epsilon}(T)$. Take the limit $P(T):=\lim _{\epsilon \rightarrow \infty} \frac{1}{\epsilon^{\alpha}} P_{\epsilon}(T)$.

4. Rewrite $P(T)$ as a linear combination of determinants.

5. Define $I_{j}=C_{j}$ as the polynomial coefficients of $P(T)$.

6. Check the functional independence of the functions $I_{j}$.

7. If necessary, symmetrize the functions $I_{j}$ in order to recover the Casimir operators of $\mathfrak{g}^{\prime}$.

The validity of the method is formally proved using the contraction explicitly. If $\Psi_{\varepsilon}$ is the automorphism of $\mathfrak{g}$ defining the contraction, then the matrix

$$
A_{k}^{\prime}=\sum_{i} \Gamma_{k}\left(X_{i}^{\prime}\right) x_{i}^{\prime}
$$

expresses the Casimir operators of $\mathfrak{g}$ over the transformed basis. Developing the corresponding characteristic polynomial $P(T)$ and taking into account the limit, after some algebraic manipulation we can obtain an expression of $P(T)$ that does not involve the contraction parameter $\epsilon$ anymore. By transitivity of contractions, the same algorithm can be applied formally to the contraction of reductive algebras.

For Lie algebras of the type $w \mathfrak{s}=\mathfrak{s} \vec{\oplus}_{\Gamma} \mathfrak{h}_{N}$, i.e., semidirect products of a simple and a Heisenberg Lie algebra, the argument remains valid for the contraction $w \mathfrak{s} \rightsquigarrow \mathfrak{s} \vec{\oplus}_{\Gamma}(2 N+1) L_{1}$. The structure of representations of simple Lie algebras compatible with Heisenberg algebras was analyzed in [6]. Depending on this structure, we obtain by contraction either an inhomogeneous Lie algebra or a general affine algebra. The first step is to compute invariants of $w \mathfrak{s}$. This is done by constructing a copy of the Levi part $\mathfrak{s}$ in the enveloping algebra of $w \mathfrak{s}^{2}$, which can be used as the simple algebra to which the preceding procedure is applied.

Remark 1. In general, the shape of $P(T)$ will depend essentially on the structure of the standard representation and the contraction considered, and possibly involves matrices depending on $T$. In particular, it will be different for the cases where this representation is by real or complex matrices, i.e., if it is of first or second genus [9]. This fact can also originate some dependence problems.

\subsection{Inhomogeneous symplectic algebras}

As an example of a general class to which the algorithm applies, we consider the inhomogeneous symplectic Lie algebras $I \mathfrak{s p}(2 N, \mathbb{R})$ and the matrix formula obtained for them in [5]. Over the basis $\left\{X_{i, j}, X_{-i, j}, X_{i, j}, P_{i}, Q_{i}\right\}(1 \leq i, j \leq N)$ the brackets of $I \mathfrak{s p}(2 N, \mathbb{R})$ are given by:

$$
\begin{aligned}
& {\left[X_{i, j}, X_{k, l}\right]=\delta_{j k} X_{i l}-\delta_{i l} X_{k j}+\varepsilon_{i} \varepsilon_{j} \delta_{j,-l} X_{k,-i}-\varepsilon_{i} \varepsilon_{j} \delta_{i,-k} X_{-j, l},} \\
& {\left[X_{i, j}, P_{k}\right]=\delta_{j k} P_{i}, \quad\left[X_{i, j}, Q_{k}\right]=-\delta_{i k} Q_{j},} \\
& {\left[X_{-i, j}, Q_{k}\right]=-\delta_{j k} P_{i}-\delta_{i k} P_{j}, \quad\left[X_{i,-j}, P_{k}\right]=\delta_{i k} Q_{j}+\delta_{k j} Q_{i} .}
\end{aligned}
$$

\footnotetext{
${ }^{1}$ By this we mean that the limit $[X, Y]_{\infty}:=\lim _{\epsilon \rightarrow \infty} \Psi_{\epsilon}^{-1}\left[\Psi_{\epsilon}(X), \Psi_{\epsilon}(Y)\right]$ exists for any $X, Y \in \mathfrak{g}$. This equation defines a Lie algebra $\mathfrak{g}^{\prime}$ called the contraction of $\mathfrak{g}$ (by $\Psi_{\epsilon}$ ).

${ }^{2}$ These variables can be found analyzing the noncentral Casimir operator of the subalgebras $\mathfrak{s}^{\prime} \vec{\oplus}_{\Gamma} \mathfrak{h}(N)$, where $\mathfrak{s}^{\prime}$ is a simple subalgebra of rank one of $\mathfrak{s}[7,8]$.
} 
Table 1. Nonisomorphic kinematical algebras in $(3+1)$ dimensions [10].

\begin{tabular}{l|ccccccccc} 
& $\mathfrak{s o}(4,1)$ & $\mathfrak{s o}(3,2)$ & $I \mathfrak{s o}(3,1)$ & $I \mathfrak{s o}(4)$ & $\mathrm{Ne}^{\exp }$ & $\mathrm{Ne}^{\mathrm{osc}}$ & Carroll & $\mathrm{G}(2)$ & Static \\
\hline$[H, \mathbf{P}]$ & $\mathbf{K}$ & $-\mathbf{K}$ & 0 & $\mathbf{K}$ & $\mathbf{K}$ & $-\mathbf{K}$ & 0 & 0 & 0 \\
{$[H, \mathbf{K}]$} & $\mathbf{P}$ & $\mathbf{P}$ & $\mathbf{P}$ & 0 & $\mathbf{P}$ & $\mathbf{P}$ & 0 & $\mathbf{P}$ & 0 \\
{$[\mathbf{P}, \mathbf{P}]$} & $\mathbf{J}$ & $-\mathbf{J}$ & 0 & $\mathbf{J}$ & 0 & 0 & 0 & 0 & 0 \\
{$[\mathbf{K}, \mathbf{K}]$} & $-\mathbf{J}$ & $-\mathbf{J}$ & $-\mathbf{J}$ & 0 & 0 & 0 & 0 & 0 & 0 \\
{$[\mathbf{P}, \mathbf{K}]$} & $H$ & $H$ & $H$ & $H$ & 0 & 0 & $H$ & 0 & 0
\end{tabular}

Proposition 1. Let $N \geq 2$. Then the Casimir operators $C_{2 k}$ of $I \mathfrak{s p}(2 N, \mathbb{R})$ are given by the coefficients of the polynomial

$$
\left|C-T \operatorname{Id}_{2 N+1}\right|+\left|C_{2 N+1,2 N+1}-T \operatorname{Id}_{2 N}\right| T=\sum_{k=1}^{N} C_{2 k+1} T^{2 N+1-2 k},
$$

where

$$
C=\left(\begin{array}{ccccccc}
x_{1,1} & \cdots & x_{1, N} & -x_{-1,1} & \cdots & -x_{-1, N} & p_{1} T \\
\vdots & & \vdots & \vdots & & \vdots & \vdots \\
x_{N, 1} & \cdots & x_{N, N} & -x_{-1, N} & \cdots & -x_{-N, N} & p_{N} T \\
x_{1,-1} & \cdots & x_{1,-N} & -x_{1,1} & \cdots & -x_{N, 1} & q_{1} T \\
\vdots & & \vdots & \vdots & & \vdots & \vdots \\
x_{1,-N} & \cdots & x_{N,-N} & -x_{1, N} & \cdots & -x_{N, N} & q_{N} T \\
-q_{1} & \cdots & -q_{N} & p_{1} & \cdots & p_{N} & 0
\end{array}\right)
$$

and $C_{2 N+1,2 N+1}$ is the minor of $C$ obtained deleting the last row and column. Moreover $\operatorname{deg} C_{2 k+1}=$ $2 k+1$.

\section{The kinematical algebras in $(3+1)$-dimensions}

As an interesting physical example, we apply the preceding procedure to the class of kinematical Lie algebras in $(3+1)$-dimensions. This choice is appropriate since it contains algebras of both types and because they are all related by contractions $[10,11]$, thus by transitivity the procedure can be applied. Although their invariants have been obtained repeatedly in different contexts [12, 13], it is worthy to be done on the basis of the above arguments, which, moreover, show that the matrix providing the invariants is not necessarily related to a faithful representation of the algebra.

Following the original notation of [10], kinematical Lie algebras are defined over the basis $\left\{J_{\alpha}, P_{\alpha}, K_{\alpha}, H\right\}_{1<\alpha<3}$, where $J_{\alpha}$ are spatial rotations, $P_{\alpha}$ spatial translations, $K_{\alpha}$ the boosts and $H$ the time translation, constrained to the condition of space isotropy

$$
\left[J_{\alpha}, J_{\beta}\right]=\varepsilon^{\alpha \beta \gamma} J_{\gamma}, \quad\left[J_{\alpha}, P_{\beta}\right]=\varepsilon^{\alpha \beta \gamma} P_{\gamma}, \quad\left[J_{\alpha}, K_{\beta}\right]=\varepsilon^{\alpha \beta \gamma} K_{\gamma}, \quad\left[J_{\alpha}, H\right]=0,
$$

as well as the assumption that time-reversal and parity are automorphisms of the group. Taking the compact notation $[\mathbf{X}, \mathbf{Y}]=\mathbf{Z}$ for $\left[X_{\alpha}, Y_{\beta}\right]=\varepsilon^{\alpha \beta \gamma} Z_{\gamma}$, the brackets of the nonisomorphic kinematical Lie algebras are given in Table $1^{3}$.

As shown in [10], any kinematical Lie algebra is obtained by contraction of the simple de Sitter Lie algebras $\mathfrak{s o}(3,2)$ and $\mathfrak{s o}(4,1)$. With the exception of the static algebra, all of the remaining ones possess two independent invariants.

\footnotetext{
${ }^{3}$ We have omitted the Para-Poincaré and Para-Galilei Lie algebras, since they are isomorphic to the Poincaré and Galilei algebras, respectively, although they are physically different. For purposes of invariants, this physical distinction is irrelevant.
} 


\subsection{De Sitter algebras}

Since both de Sitter algebras are simple and pseudo-orthogonal, their Casimir operators follow at once from application of the Gel'fand formula. For obtaining the invariants over the kinematical basis above, the matrix (1) has to be slightly transformed.

1. Anti de Sitter algebra $\mathfrak{s o}(3,2)$. The matrix related to the standard representations is:

$$
D=\left(\begin{array}{ccccc}
0 & j_{3} & j_{2} & -k_{1} & p_{1} \\
-j_{3} & 0 & j_{1} & k_{2} & -p_{2} \\
-j_{2} & -j_{1} & 0 & -k_{3} & p_{3} \\
-k_{1} & k_{2} & -k_{3} & 0 & h \\
p_{1} & -p_{2} & p_{3} & -h & 0
\end{array}\right)
$$

Computing the characteristic polynomial we have $\left|D-T \operatorname{Id}_{5}\right|=T^{5}+C_{2} T^{3}+C_{4} T$, where

$$
\begin{aligned}
& C_{2}=j_{\alpha} j^{\alpha}-p_{\alpha} p^{\alpha}-k_{\alpha} k^{\alpha}+h^{2}, \\
& C_{4}=j_{\alpha} j^{\alpha} h^{2}+\left(\left(p_{\alpha} p^{\alpha}\right)\left(k_{\alpha} k^{\alpha}\right)-\left(p_{\alpha} k^{\alpha}\right)^{2}\right)-\left(j_{\alpha} p^{\alpha}\right)^{2}-\left(j_{\alpha} k^{\alpha}\right)^{2}-2 \varepsilon^{\alpha \beta \gamma} j_{\alpha} p_{\beta} k_{\gamma} h .
\end{aligned}
$$

2. De Sitter algebra $\mathfrak{s o}(4,1)$. The resulting matrix is similar to the previous one:

$$
D=\left(\begin{array}{ccccc}
0 & j_{3} & j_{2} & -k_{1} & p_{1} \\
-j_{3} & 0 & j_{1} & k_{2} & -p_{2} \\
-j_{2} & -j_{1} & 0 & -k_{3} & p_{3} \\
-k_{1} & k_{2} & -k_{3} & 0 & h \\
-p_{1} & p_{2} & -p_{3} & h & 0
\end{array}\right)
$$

Then we have $\left|D-T \operatorname{Id}_{5}\right|=T^{5}+C_{2} T^{3}+C_{4} T$, where

$$
\begin{aligned}
& C_{2}=j_{\alpha} j^{\alpha}+p_{\alpha} p^{\alpha}-k_{\alpha} k^{\alpha}-h^{2}, \\
& C_{4}=-j_{\alpha} j^{\alpha} h^{2}-\left(\left(p_{\alpha} p^{\alpha}\right)\left(k_{\alpha} k^{\alpha}\right)-\left(p_{\alpha} k^{\alpha}\right)^{2}\right)+\left(j_{\alpha} p^{\alpha}\right)^{2}-\left(j_{\alpha} k^{\alpha}\right)^{2}+2 \varepsilon^{\alpha \beta \gamma} j_{\alpha} p_{\beta} k_{\gamma} h .
\end{aligned}
$$

For these two algebras, the result follows at once from the Gel'fand formula.

\subsection{The nonrelativistic cosmological Lie algebras}

The Newton algebras $\mathrm{Ne}^{+}$and $\mathrm{Ne}^{-}$are obtained as contractions of the de Sitter and Anti de Sitter algebras, respectively. Since the subalgebra generated by $\left\{K_{\alpha}, P_{\alpha}\right\}$ is Abelian, it will follow that the invariants of these algebras will not depend on the rotation and time-translation generators.

1. The Newton algebra $\mathrm{Ne}^{-}$[oscillating universe]. It can be easily verified that this Lie algebra is indeed an extension by a derivation of the nine dimensional Lie algebra $\mathfrak{s o}(3) \vec{\oplus}_{2 a d \mathfrak{s o}(3)} 6 L_{1}$. By the comment above, the matrix giving the Casimir operators of $\mathrm{Ne}^{-}$will not be related to a faithful representation of the algebra. We have:

$$
D=\left(\begin{array}{ccccc}
0 & 0 & 0 & -k_{1} & p_{1} \\
0 & 0 & 0 & k_{2} & -p_{2} \\
0 & 0 & 0 & -k_{3} & p_{3} \\
-k_{1} & k_{2} & -k_{3} & 0 & 0 \\
p_{1} & -p_{2} & p_{3} & 0 & 0
\end{array}\right)
$$

Expanding the secular equation we arrive at $\left|D-T \operatorname{Id}_{5}\right|=T^{5}+C_{2} T^{3}+C_{4} T$, where

$$
\begin{aligned}
& C_{2}=-p_{\alpha} p^{\alpha}-k_{\alpha} k^{\alpha}, \\
& C_{4}=\left(\left(p_{\alpha} p^{\alpha}\right)\left(k_{\alpha} k^{\alpha}\right)-\left(p_{\alpha} k^{\alpha}\right)^{2}\right) .
\end{aligned}
$$


2. The Newton algebra $\mathrm{Ne}^{+}$[expanding universe]. This Lie algebra is also an extension by a derivation of $\mathfrak{s o}(3) \vec{\oplus}_{2 a d \mathfrak{s o}(3)} 6 L_{1}$. In this case the matrix to be used is:

$$
D=\left(\begin{array}{ccccc}
0 & 0 & 0 & -k_{1} & p_{1} \\
0 & 0 & 0 & k_{2} & -p_{2} \\
0 & 0 & 0 & -k_{3} & p_{3} \\
-k_{1} & k_{2} & -k_{3} & 0 & 0 \\
-p_{1} & p_{2} & -p_{3} & 0 & 0
\end{array}\right)
$$

Then we have $\left|D-T \operatorname{Id}_{5}\right|=T^{5}+C_{2} T^{3}+C_{4} T$, where

$$
\begin{aligned}
& C_{2}=p_{\alpha} p^{\alpha}-k_{\alpha} k^{\alpha}, \\
& C_{4}=\left(\left(p_{\alpha} p^{\alpha}\right)\left(k_{\alpha} k^{\alpha}\right)-\left(p_{\alpha} k^{\alpha}\right)^{2}\right) .
\end{aligned}
$$

\subsection{The inhomogeneous (pseudo)-orthogonal algebras}

In order to obtain the matrix for the inhomogeneous algebras $I \mathfrak{s o}(3,1)$ and $I \mathfrak{s o}(4)$, we use the contractions $\mathfrak{s o}(3,2) \rightsquigarrow I \mathfrak{s o}(3,1)$ and $\mathfrak{s o}(4,1) \rightsquigarrow I \mathfrak{s o}(4)$, respectively.

1. The Poincaré Lie algebra $I \mathfrak{s o}(3,1)$. The matrix $D$ is given by

$$
D=\left(\begin{array}{ccccc}
0 & j_{3} & j_{2} & -k_{1} & p_{1} \\
-j_{3} & 0 & j_{1} & k_{2} & -p_{2} \\
-j_{2} & -j_{1} & 0 & -k_{3} & p_{3} \\
-k_{1} & k_{2} & -k_{3} & 0 & h \\
p_{1} & -p_{2} & p_{3} & -h & 0
\end{array}\right)
$$

We define $P(T)=\left|D-T \operatorname{Id}_{5}\right|+T\left|D_{55}-T \operatorname{Id}_{4}\right|$, where $D_{55}$ is the minor of $D$ obtained by deleting the fifth column and row. In particular, the matrix $D_{55}$ corresponds to that of the Lorentz algebra $\mathfrak{s o}(3,1)$. Expanding the expression for $P(T)$, we get $P(T)=$ $T^{5}+C_{2} T^{3}+C_{4} T$, where

$$
\begin{aligned}
& C_{2}=h^{2}-p_{\alpha} p^{\alpha}, \\
& C_{4}=j_{\alpha} j^{\alpha} h^{2}+\left(p_{\alpha} p^{\alpha}\right)\left(k_{\alpha} k^{\alpha}\right)-\left(j_{\alpha} p^{\alpha}\right)^{2}-\left(p_{\alpha} k^{\alpha}\right)^{2}-2 \varepsilon^{\alpha \beta \gamma} j_{\alpha} p_{\beta} k_{\gamma} h .
\end{aligned}
$$

Moreover, the matrix $D$ can be decomposed as

$$
D=D_{1}+D_{2}=\left(\begin{array}{ccccc}
0 & j_{3} & j_{2} & -k_{1} & p_{1} \\
-j_{3} & 0 & j_{1} & k_{2} & -p_{2} \\
-j_{2} & -j_{1} & 0 & -k_{3} & p_{3} \\
-k_{1} & k_{2} & -k_{3} & 0 & h \\
0 & 0 & 0 & 0 & 0
\end{array}\right)+\left(\begin{array}{ccccc}
0 & 0 & 0 & 0 & 0 \\
0 & 0 & 0 & 0 & 0 \\
0 & 0 & 0 & 0 & 0 \\
0 & 0 & 0 & 0 & 0 \\
p_{1} & -p_{2} & p_{3} & -h & 0
\end{array}\right)
$$

where $D_{1}$ defines a faithful representation of $I \mathfrak{s o}(3,1)$.

2. The inhomogeneous algebra $I \mathfrak{s o}$ (4). Here the polynomial is $P(T)=\left|D-T \operatorname{Id}_{5}\right|+T \mid D_{55}-$ $T \mathrm{Id}_{4} \mid$, where $D_{55}$ is the minor of $D$ obtained deleting the fifth column and row. The matrix $D$ is given by

$$
D=\left(\begin{array}{ccccc}
0 & j_{3} & j_{2} & -k_{1} & p_{1} \\
-j_{3} & 0 & j_{1} & k_{2} & -p_{2} \\
-j_{2} & -j_{1} & 0 & -k_{3} & p_{3} \\
-k_{1} & k_{2} & -k_{3} & 0 & h \\
-p_{1} & p_{2} & -p_{3} & h & 0
\end{array}\right)
$$


Expanding $P(T)$, we get $P(T)=T^{5}+C_{2} T^{3}+C_{4} T$, with

$$
\begin{aligned}
& C_{2}=-h^{2}-k_{\alpha} k^{\alpha}, \\
& C_{4}=j_{\alpha} j^{\alpha} h^{2}+\left(p_{\alpha} p^{\alpha}\right)\left(k_{\alpha} k^{\alpha}\right)+\left(j_{\alpha} k^{\alpha}\right)^{2}-\left(p_{\alpha} k^{\alpha}\right)^{2}-2 \varepsilon^{\alpha \beta \gamma} j_{\alpha} p_{\beta} k_{\gamma} h .
\end{aligned}
$$

In this case, $D$ decomposes as

$$
D=D_{1}+D_{2}=\left(\begin{array}{ccccc}
0 & j_{3} & j_{2} & 0 & p_{1} \\
-j_{3} & 0 & j_{1} & 0 & -p_{2} \\
-j_{2} & -j_{1} & 0 & 0 & p_{3} \\
-k_{1} & k_{2} & -k_{3} & 0 & h \\
-p_{1} & p_{2} & -p_{3} & 0 & 0
\end{array}\right)+\left(\begin{array}{ccccc}
0 & 0 & 0 & -k_{1} & 0 \\
0 & 0 & 0 & k_{2} & 0 \\
0 & 0 & 0 & -k_{3} & 0 \\
0 & 0 & 0 & 0 & 0 \\
0 & 0 & 0 & h & 0
\end{array}\right)
$$

and $D_{1}$ is the matrix related to a the faithful representation of $I \mathfrak{s o}(4)$ by $5 \times 5$ matrices.

\subsection{The Carroll Lie algebra}

Among the classical kinematical Lie algebras, the Carroll Lie algebra is the only isomorphic to the semidirect product of a simple Lie algebra (the compact algebra $\mathfrak{s o}(3)$ ) and a Heisenberg Lie algebra. Indeed the noncentral Casimir operator can be determined using the determinant procedure developed in [8]. However, the Casimir operators (the second is trivial, since the centre is nonzero) can also be obtained by the same method as before.

We define $P(T)=\left|D-T \operatorname{Id}_{5}\right|$, where $D$ is the matrix

$$
D=\left(\begin{array}{ccccc}
0 & j_{3} & j_{2} & -k_{1} & p_{1} \\
-j_{3} & 0 & j_{1} & k_{2} & -p_{2} \\
-j_{2} & -j_{1} & 0 & -k_{3} & p_{3} \\
-k_{1} & k_{2} & -k_{3} & T & h \\
-p_{1} & p_{2} & -p_{3} & h & T
\end{array}\right)
$$

Observe that in this case, the matrix $D$ is dependent on the variable $T$. Expanding, we get $P(T)=T^{5}+C_{2} T^{3}+C_{4} T$, where

$$
\begin{aligned}
& C_{2}=h^{2}, \\
& C_{4}=j_{\alpha} j^{\alpha} h^{2}+\left(p_{\alpha} p^{\alpha}\right)\left(k_{\alpha} k^{\alpha}\right)-\left(p_{\alpha} k^{\alpha}\right)^{2}-2 \varepsilon^{\alpha \beta \gamma} j_{\alpha} p_{\beta} k_{\gamma} h .
\end{aligned}
$$

The matrix $D$ decomposes in this case as

$$
D=D_{1}+D_{2}=\left(\begin{array}{ccccc}
0 & j_{3} & j_{2} & 0 & p_{1} \\
-j_{3} & 0 & j_{1} & 0 & -p_{2} \\
-j_{2} & -j_{1} & 0 & 0 & p_{3} \\
-k_{1} & k_{2} & -k_{3} & 0 & h \\
0 & 0 & 0 & 0 & 0
\end{array}\right)+\left(\begin{array}{ccccc}
0 & 0 & 0 & -k_{1} & 0 \\
0 & 0 & 0 & k_{2} & 0 \\
0 & 0 & 0 & -k_{3} & 0 \\
0 & 0 & 0 & T & 0 \\
-p_{1} & p_{2} & -p_{3} & h & T
\end{array}\right)
$$

Again, the matrix $D_{1}$ gives rise to a faithful representation of the Carroll algebra.

\subsection{The Galilei algebra $G(2)$}

As happened for the Newton algebras, the Casimir operators of the Galilei algebra do not depend on the variables $\left\{j_{\alpha}, h\right\}$. Here we consider the polynomial $P(T)=\left|D-T \operatorname{Id}_{5}\right|$, where

$$
D=\left(\begin{array}{ccccc}
0 & 0 & 0 & -k_{1} & p_{1} \\
0 & 0 & 0 & k_{2} & -p_{2} \\
0 & 0 & 0 & -k_{3} & p_{3} \\
-k_{1} & k_{2} & -k_{3} & 0 & 0 \\
-p_{1} & p_{2} & -p_{3} & 0 & T
\end{array}\right)
$$


Also in this case, the matrix $D$ is dependent on the variable $T$. Developing the polynomial we obtain $P(T)=T^{5}+C_{2} T^{3}+C_{4} T$, where

$$
\begin{aligned}
& C_{2}=p_{\alpha} p^{\alpha}, \\
& C_{4}=\left(p_{\alpha} p^{\alpha}\right)\left(k_{\alpha} k^{\alpha}\right)-\left(p_{\alpha} k^{\alpha}\right)^{2} .
\end{aligned}
$$

By the remark above, the preceding matrix is not related to a faithful representation of the algebra.

\subsection{The static Lie algebra}

This algebra is nothing but the splittable affine Lie algebra $\left(\mathfrak{s o}(3) \vec{\oplus}_{2 a d \mathfrak{s o}(3)} 6 L_{1}\right) \oplus \mathbb{R}$. As commented, it has four invariants, all of the degree two,

$$
I_{1}=h, \quad I_{2}=p_{\alpha} p^{\alpha}, \quad I_{3}=k_{\alpha} k^{\alpha}, \quad I_{5}=k_{\alpha} p^{\alpha} .
$$

To obtain them in matrix form, we consider

$$
D=\left(\begin{array}{ccccc}
0 & 0 & 0 & -k_{1} & p_{1} T \\
0 & 0 & 0 & k_{2} & -p_{2} T \\
0 & 0 & 0 & -k_{3} & p_{3} T \\
-k_{1} & k_{2} & -k_{3} & 0 & -h T \\
-p_{1} & p_{2} & -p_{3} & -h & 0
\end{array}\right)
$$

and obtain $P(T)=T^{5}+\left(I_{2}-I_{1}^{2}\right) T^{4}-I_{3} T^{3}-\left(I_{2} I_{3}-I_{5}^{2}\right) T^{2}$. Simplifyng the coefficients we recover the basis of invariants above. Since the variables associated to the rotations $J_{\alpha}$ do not appear in the invariants, the matrix $D$ does not provide a representation of the static algebra.

For later use we consider the following functions:

$$
\begin{aligned}
& I_{1}=h, \quad I_{2}=p_{\alpha} p^{\alpha}, \quad I_{3}=k_{\alpha} k^{\alpha}, \quad I_{4}=j_{\alpha} j^{\alpha}, \quad I_{5}=k_{\alpha} p^{\alpha}, \\
& I_{6}=j_{\alpha} k^{\alpha}, \quad I_{7}=j_{\alpha} p^{\alpha}, \quad M=\varepsilon^{\alpha \beta \gamma} j_{\alpha} p_{\beta} k_{\gamma} .
\end{aligned}
$$

\section{Applications: The missing label problem}

As known, irreducible representations of a semisimple Lie algebra are labelled unambigously by the eigenvalues of Casimir operators. In a more general frame, irreducible representations of a Lie algebra $\mathfrak{g}$ are labelled usingby means of the eigenvalues of its generalized Casimir invariants $[14,15]$. The number of internal labels needed is

$$
i=\frac{1}{2}(\operatorname{dim} \mathfrak{g}-\mathcal{N}(\mathfrak{g})) .
$$

If we use a subalgebra $\mathfrak{h}$ label the basis states of $\mathfrak{g}$, then we obtain $\frac{1}{2}(\operatorname{dim} \mathfrak{h}+\mathcal{N}(\mathfrak{h}))+l^{\prime}$ labels from $\mathfrak{h}$, where $l^{\prime}$ is the number of invariants of $\mathfrak{g}$ that depend only on variables of the subalgebra $\mathfrak{h}[15]$. In order to label irreducible representations of $\mathfrak{g}$ uniquely, it is therefore necessary to find

$$
n=\frac{1}{2}(\operatorname{dim} \mathfrak{g}-\mathcal{N}(\mathfrak{g})-\operatorname{dim} \mathfrak{h}-\mathcal{N}(\mathfrak{h}))+l^{\prime}
$$

additional operators, which are usually called missing label operators. They are found by integrating the equations of system (2) corresponding to the subalgebra generators. The total number of available operators of this kind is easily shown to be $m=2 n$. 
In this situation, it is plausible to think that whenever the Casimir operators of a Lie algebra $\mathfrak{g}$ can be determined using determinants of (polynomial) matrices, the same procedure could hold for computing missing label operators according to some subalgebra $\mathfrak{h}$. In this section we analyze the missing label problem for the chain

$$
\mathfrak{s o}(3) \hookrightarrow \mathfrak{g},
$$

where $\mathfrak{g}$ is a kinematical Lie algebra in $(3+1)$-dimensions and $\mathfrak{s o}(3)$ the compact subalgebra generated by the $\left\{J_{\mu \nu}\right\}$. The missing label operators are among the solutions of the equations:

$$
\begin{aligned}
& j_{3} \frac{\partial F}{\partial j_{2}}-j_{2} \frac{\partial F}{\partial j_{3}}+p_{3} \frac{\partial F}{\partial p_{2}}-p_{2} \frac{\partial F}{\partial p_{3}}+k_{3} \frac{\partial F}{\partial k_{2}}-k_{2} \frac{\partial F}{\partial k_{3}}=0 \\
& -j_{3} \frac{\partial F}{\partial j_{1}}+j_{1} \frac{\partial F}{\partial j_{3}}-p_{3} \frac{\partial F}{\partial p_{1}}+p_{1} \frac{\partial F}{\partial p_{3}}-k_{3} \frac{\partial F}{\partial k_{1}}+k_{1} \frac{\partial F}{\partial k_{3}}=0 \\
& j_{2} \frac{\partial F}{\partial j_{1}}-j_{1} \frac{\partial F}{\partial j_{2}}+p_{2} \frac{\partial F}{\partial p_{1}}-p_{1} \frac{\partial F}{\partial p_{2}}+k_{2} \frac{\partial F}{\partial k_{1}}-k_{1} \frac{\partial F}{\partial k_{2}}=0 .
\end{aligned}
$$

Due to the space isotropy condition, the above system is valid for any kinematical Lie algebra. According to formula (6), there are

$$
n=\frac{1}{2}(\operatorname{dim} \mathfrak{g}-\mathcal{N}(\mathfrak{g})-\operatorname{dim} \mathfrak{s o}(3)-\mathcal{N}(s o(3)))+l^{\prime}=\frac{1}{2}(6-\mathcal{N}(\mathfrak{g}))+l^{\prime}
$$

missing labels. In any case we have $l^{\prime}=0$. Moreover, for any kinematical algebra, with the exception of the static algebra, we obtain $n=2$ and four available missing label operators, while for the static algebra we get $n=1$ and $m=2$. By using of the matrix notation, the system can be rewritten as:

$$
\left(\begin{array}{cccccccccc}
0 & j_{3} & -j_{2} & 0 & p_{3} & -p_{2} & 0 & k_{3} & -k_{2} & 0 \\
-j_{3} & 0 & j_{1} & -p_{3} & 0 & p_{1} & -k_{3} & 0 & k_{1} & 0 \\
j_{2} & -j_{1} & 0 & p_{2} & -p_{1} & 0 & k_{2} & -k_{1} & 0 & 0
\end{array}\right)\left(\begin{array}{c}
\frac{\partial F}{\partial j_{\alpha}} \\
\frac{\partial F}{\partial p_{\alpha}} \\
\frac{\partial F}{\partial k_{\alpha}} \\
\frac{\partial F}{\partial h}
\end{array}\right)=0 .
$$

Since the matrix has rank three, there are seven independent solutions of the system. The number of solutions that do not depend on the variables $\left\{j_{\alpha}\right\}$ of the subalgebra $\mathfrak{s o}(3)$ is given by [16]:

$$
\mathcal{N}^{\prime}=7-\operatorname{rank}\left(\begin{array}{ccccccc}
0 & p_{3} & -p_{2} & 0 & k_{3} & -k_{2} & 0 \\
-p_{3} & 0 & p_{1} & -k_{3} & 0 & k_{1} & 0 \\
p_{2} & -p_{1} & 0 & k_{2} & -k_{1} & 0 & 0
\end{array}\right)=4 .
$$

It is straightforward to verify that a complete system of independent solutions is given by:

$$
\left\{I_{1}=h, I_{2}=p_{\alpha} p^{\alpha}, I_{3}=k_{\alpha} k^{\alpha}, I_{4}=j_{\alpha} j^{\alpha}, I_{5}=k_{\alpha} p^{\alpha}, I_{6}=j_{\alpha} k^{\alpha}, I_{7}=j_{\alpha} p^{\alpha}\right\} .
$$

In particular, the invariants $I_{1}, I_{2}, I_{3}, I_{5}$, which are the independent solutions not involving the variables $j_{\alpha}$, constitute a set of solutions for the static Lie algebra. The function $M=\varepsilon^{\alpha \beta \gamma} j_{\alpha} p_{\beta} k_{\gamma}$ of (5) is functionally dependent on the previous functions, as shown by the relation

$$
M^{2}=I_{5}^{2} I_{4}+I_{7}^{2} I_{3}-I_{6}^{2} I_{2}-I_{2} I_{3} I_{4}-2 I_{5} I_{6} I_{7} .
$$

To see how the matrices used for the Casimir operators of kinematical algebras can also be applied to the MLP, we consider the Anti de Sitter algebra $\mathfrak{s o}(3,2)$. In the notation of $(8)$, the 
Table 2. Missing label operators for the chain $\mathfrak{s o}(3) \hookrightarrow \mathfrak{g}$.

\begin{tabular}{|c|c|c|c|}
\hline Algebra $\mathfrak{g}$ & Casimir operators of $\mathfrak{g}$ & $\begin{array}{l}\text { Missing label } \\
\text { operators }\end{array}$ & $\begin{array}{l}\text { MLP obtained from } \\
\text { the reduced matrix }\end{array}$ \\
\hline $\mathfrak{s o}(4,1)$ & $\begin{array}{l}I_{1}^{2}-I_{2}+I_{3}-I_{4} \\
I_{1}^{2} I_{4}+I_{2} I_{3}-I_{5}^{2}+I_{6}^{2}-I_{7}^{2}-2 I_{1} M\end{array}$ & $\left\{I_{2}, I_{3}, I_{5}, I_{6}\right\}$ & $\left\{I_{2}, I_{3}, I_{5}\right\}$ \\
\hline $\mathfrak{s o}(3,2)$ & $\begin{array}{l}I_{1}^{2}-I_{2}-I_{3}+I_{4} \\
I_{1}^{2} I_{4}+I_{2} I_{3}-I_{5}^{2}-I_{6}^{2}-I_{7}^{2}-2 I_{1} M\end{array}$ & $\left\{I_{2}, I_{3}, I_{5}, I_{6}\right\}$ & $\left\{I_{2}, I_{3}, I_{5}\right\}$ \\
\hline$I \mathfrak{s o}(3,1)$ & $\begin{array}{l}I_{1}^{2}-I_{2} \\
I_{1}^{2} I_{4}+I_{2} I_{3}-I_{5}^{2}-I_{7}^{2}-2 I_{1} M\end{array}$ & $\left\{I_{2}, I_{3}, I_{5}, I_{7}\right\}$ & $\left\{I_{2}, I_{3}, I_{5}\right\}$ \\
\hline$I \mathfrak{s o}(4)$ & $\begin{array}{l}I_{1}^{2}+I_{2} \\
I_{1}^{2} I_{4}+I_{2} I_{3}-I_{5}^{2}+I_{6}^{2}-2 I_{1} M\end{array}$ & $\left\{I_{2}, I_{3}, I_{5}, I_{6}\right\}$ & $\left\{I_{2}, I_{3}, I_{5}\right\}$ \\
\hline $\mathrm{Ne}^{+}$ & $\begin{array}{l}I_{2}-I_{3} \\
I_{2} I_{3}-I_{5}^{2}\end{array}$ & $\left\{I_{1}, I_{2}, I_{6}, I_{7}\right\}$ & $\left\{I_{2}\right\}$ \\
\hline $\mathrm{Ne}^{-}$ & $\begin{array}{l}I_{2}+I_{3} \\
I_{2} I_{3}-I_{5}^{2}\end{array}$ & $\left\{I_{1}, I_{2}, I_{6}, I_{7}\right\}$ & $\left\{I_{2}\right\}$ \\
\hline Carroll & $\begin{array}{l}I_{1}^{2} \\
I_{1}^{2} I_{4}+I_{2} I_{3}-I_{5}^{2}-2 I_{1} M\end{array}$ & $\left\{I_{2}, I_{3}, I_{5}, I_{6}\right\}$ & $\left\{I_{2} I_{3}\right\}$ \\
\hline Galilei & $\begin{array}{l}I_{2} \\
I_{2} I_{3}-I_{5}^{2}\end{array}$ & $\left\{I_{1}, I_{3}, I_{6}, I_{7}\right\}$ & $\left\{I_{2} I_{3}-I_{5}^{2}\right\}$ \\
\hline Static & $I_{1}, I_{2}, I_{3}, I_{5}$ & $\left\{I_{6}, I_{7}\right\}$ & - \\
\hline
\end{tabular}

invariants of the algebra are given by $C_{2}=I_{1}^{2}-I_{2}-I_{3}+I_{4}$ and $C_{4}=I_{1}^{2} I_{4}+I_{2} I_{3}-I_{5}^{2}-I_{6}^{2}-I_{7}^{2}-$ $2 I_{1} M$, while $I_{4}$ clearly represents the Casimir operator of $\mathfrak{s o}(3)$. We now look for those missing label operators that depend only on the variables $\left\{p_{\alpha}, k_{\alpha}, h\right\}$. To this extent, we consider the matrix (4) used to compute $C_{2}$ and $C_{4}$ and replace the variables $j_{\alpha}$ by 0 . We obtain

$$
D^{\prime}=\left(\begin{array}{ccccc}
0 & 0 & 0 & -k_{1} & p_{1} \\
0 & 0 & 0 & k_{2} & -p_{2} \\
0 & 0 & 0 & -k_{3} & p_{3} \\
-k_{1} & k_{2} & -k_{3} & 0 & h \\
p_{1} & -p_{2} & p_{3} & -h & 0
\end{array}\right) .
$$

Considering the characteristic polynomial we have

$$
P(T)=\left|D^{\prime}-T \operatorname{Id}_{5}\right|=T^{5}+\left(I_{1}^{2}-I_{2}-I_{3}\right) T^{3}+\left(I_{2} I_{3}-I_{5}^{2}\right) T .
$$

It can be easily verified that $\left\{I_{2}, I_{3}, I_{5}\right\}$ are solutions of (7) independent of $\left\{C_{2}, C_{4}, I_{4}\right\}$, while $I_{1}^{2}$ is not an independent solution. Therefore the matrix $D^{\prime}$ provides three of the four available missing label operators. The fourth, which can be chosen as $I_{6}$, cannot be obtained using $D^{\prime}$, since it depends on the rotation generators.

A similar argument can be used for the remaining kinematical algebras $\mathfrak{g}$. We consider the matrix giving the invariants of $\mathfrak{g}$ and replace the subalgebra generators $j_{\alpha}$ by 0 . Then we compute the corresponding characteristic polynomial and see how many independent solutions from $\left\{C_{2}, C_{4}, I_{4}\right\}$ are obtained, where $C_{2}$ and $C_{4}$ are the quadratic and fourth order Casimir operators of $\mathfrak{g}$, respectively. Only for the static Lie algebra this method of generating missing label operators fails, since their Casimir operators are a maximal set of solutions of system (7) not depending on the $j_{\alpha}$. The corresponding results are presented in Table 2 .

\section{Final remarks}

The approach presented here to compute Casimir invariants of Lie algebras tries to extend the classical results established for semisimple algebras to their contractions, making use of the 
standard representation of the contracted algebra. Although it provides in many cases closed formulae of the invariants of contractions, the application of the Gel'fand formula is certainly only of interest when the contraction has the same number of invariants than the original algebra. Although in the algebras analyzed here no dependence problems have been encountered, one of the unsolved problems is to find sufficiency criteria to ensure that the contraction of independent invariants of an algebra provides also independent operators of the contraction. Work in this direction is in progress.

As concerns applications, we have seen that the MLP can be analyzed via the generalization of the Gel'fand method, by simply reducing the matrices by zeros corresponding to generators of the subalgebra considered. This point of view could also be interesting in combination with problems in symmetries of differential equations related to contractions of Lie algebras, such as the separation of variables [17].

\section{Acknowledgements}

The author wishes to express his gratitude to J. Lôhmus for drawing his attention to reference [12] and useful comments, and to the referee for multiple suggestions that helped to improve the manuscript. This work was supported by the research grant PR1/05-13283 of the UCM.

[1] Gel'fand I.M., The center of an infinitesimal group ring, Mat. Sbornik, 1950, V.26, 103-112.

[2] Perelomov A.M., Popov V.S., Casimir operators for semisimple Lie groups, Izv. Akad. Nauk SSSR Ser. Mat., 1968, V.32, 1368-1390.

[3] Barannik L.F., Fushchich W.I., Casimir operators for the generalized Poincaré and Galilei groups, in Proceedings of the Third International Seminar "Group Theoretical Methods in Physics" (May 22-24, 1985, Yurmala), Moscow, Nauka, 1986, 176-183.

[4] Patera J., Sharp R.T., Winternitz P., Zassenhaus H., Continuous subgroups of physics III. The de Sitter groups, J. Math. Phys., 1977, V.18, 2259-2288.

[5] Campoamor-Stursberg R., A new matrix method for the Casimir operators of the Lie algebras $w \mathfrak{s p}(N, \mathbb{R})$ and $I \mathfrak{s p}(2 N, \mathbb{R})$, J. Phys. A: Math. Gen., 2005, V.38, 4187-4208.

[6] Campoamor-Stursberg R., Über die Struktur der Darstellungen komplexer halbeinfacher Lie-Algebren, die mit einer Heisenberg-Algebra verträglich sind, Acta Phys. Polon. B, 2005, V.36, 2869-2886.

[7] Quesne C., Casimir operators of semidirect sum Lie algebras, J. Phys. A: Math. Gen., 1988, V.21, L321L324.

[8] Campoamor-Stursberg R., Intrinsic formulae for the Casimir operators of semidirect products of the exceptional Lie algebra $G_{2}$ and a Heisenberg Lie algebra, J. Phys. A: Math. Gen., 2004, V.37, 9451-9466.

[9] Iwahori N., On real irreducible representations of Lie algebras, Nagoya Math. J., 1959, V.14, 59-83.

[10] Bacry H., Lévy-Leblond J.M., Possible kinematics, J. Math. Phys., 1968, V.9, 1605-1614.

[11] Lôhmus J., Tammelo R., Contractions and deformations of space-time algebras I, Hadronic J., 1997, V.20, 361-416.

[12] Gromov N.A., Contractions and analytic continuations of the classical groups. A unified approach, Syktyvkar, Akad. Nauk SSSR Ural. Otdel., 1990 (in Russian).

[13] Herranz F.J., Santander M., Casimir invariants for the complete family of quasisimple orthogonal Lie algebras, J. Phys. A: Math. Gen, 1997, V.30, 5411-5426; physics/9702032.

[14] Sharp R.T., Internal-labelling operators, J. Math. Phys., 1975, V.16, 2050-2053.

[15] Peccia A., Sharp R.T., Number of independent missing label operators, J. Math. Phys., 1976, V.17, 13131314.

[16] Campoamor-Stursberg R., The structure of the invariants of perfect Lie algebras, J. Phys. A: Math. Gen., 2003, V.36, 6709-6723.

[17] Winternitz P., Izmest'ev A.A., Pogosyan G.S., Sissakian A.N., Contractions of Lie algebras and separation of variables, Fiz. Elementar. Chastits i Atom. Yadra, 2001, V.32, 84-87. 